\title{
SIMULATION MODELLING OF A COMPANY PROVIDING TWO QUALITATIVELY DIFFERENT SERVICES TO MARKET
}

\author{
Runje, B. ; Stepanic, J.; Mihaljevic, M.*; Horvatic, A.* \& Kondic, V. ${ }^{* *}$ \\ ${ }^{*}$ University of Zagreb - Faculty of Mechanical Engineering and Naval Architecture, Zagreb, Croatia \\ ${ }^{* *}$ University North - University Center Varaždin, Varaždin, Croatia \\ E-Mail: biserka.runje@fsb.hr, josip.j.stepanic@fsb.hr, morana.mihaljevic@fsb.hr, \\ amalija.horvatic@fsb.hr, veljko.kondic@unin.hr
}

\begin{abstract}
Simulation modelling is applied onto a market dynamics of a company conducting two qualitatively different services and has a bounded total daily volume. The model contains qualitatively and in a simplified form the characteristics of the dynamics of a general service providing company. The company applies a single strategy how to influence, thus minimise, the number of agents who wait for a given service. The strategy is realised by changing the relative proportion of the daily conducted services. Rather small modification of priority level between the two services influences considerably both the number and the type of end users who wait for a particular service. The model enables the researchers to test different strategies how to control the number of end users who wait for services, by proper changes in priority level of services conducted. The fact important for practitioners is that partial optimisation is achieved through slight difference in priorities between the services.

(Received in October 2015, accepted in January 2016. This paper was with the authors 1 week for 1 revision.)
\end{abstract}

Key Words: Simulation Modelling, Service Providing Company, Adaptation, Market Demand

\section{INTRODUCTION}

\subsection{Nature of the problem}

Optimisation of market dynamics for some service providing company is a valuable, yet complex task. Its complexity arises from the generally significant market competition, from relatively large number of stakeholders with generally non-congruent attitudes, from the high quality level which potential customers expect, as well as from all the underlying and intertwined processes that needs to be conducted in a harmonised way.

Simulation modelling [1] arose as a method that has been enabling the company's management, stakeholders or any interested researchers to extract the optimised ways of market dynamics in case of a service providing company.

\subsection{Previous work}

Simulation modelling has been applied to a diverse types of services, ranging from mass to uniquely performed services, with examples covering general approaches [2-4], broadcasting [5], laboratory evaluations [6-8], or health services [9-11], to list a few.

In particular, Baxter et al. contributed to a general approach by using agent-based modelling to formulate and validate the Customer Relationship Management [2]. In that approach the customer population is profiled. The authors analyse spreading of information about the company among the population of customers and potential customers. Overall, Baxter et al. demonstrate the higher quality of their approach in comparison with approaches utilising macroscopic modelling with averaged data about (potential) customers. Kaihara analyses supply chain [3], as comprising several elements of complete service, in case of a highly dynamic environment by which presumably the customer population is meant. It is shown in [3] that, after taking into account the interactions between the customers, one reaches 
the optimal distribution for allocating the product between the production and selling [3]. Runje et al. formulate a general, market-centred simulation of a company [4], corresponding to a simplified agent-based model. It includes a variety of market (i.e. customers) characteristics as well as a number of possible company realisations in a way that makes possible comparison of its influence in duration and intensity. Terano and Naitoh formulate the agent-based model to extract the optimal market strategy in a modelled market [5]. Importance in their approach is given to the competition among companies. The companies optimise their functioning based on the collected, time dependent data. They validated their results for the markets of television programme and of audio-cassettes [5]. Acko et al. [6, 7] and Crepinsek Lipus et al. [8] concentrate on modelling of the testing laboratories. They focus on the activities that form the services which a testing laboratory provides to a highly specialised market and emphasize its relations to environment, i.e. the customer population. These relations are strictly prescribed by regulatory institutions, thus are not formulated gradually and spontaneously by customers. In that way a part of the environment complexity is absorbed and henceforth the market dynamics simplified which in consequence makes possible reaching correspondingly higher quality levels by testing laboratories, as demonstrated in [7]. Health services are somewhat different from previous examples because of probably the highest average level of end user involvement. That eventually brings about the extreme complexity of health services, realised in the large number of significant variables to be traced when evaluating the services, and realised in the observed fluctuations of end user's evaluation of provided services. In particular, Williams et al. tend to formulate the absolute meaning of the questionnaires that end user fills about the given service [9], motivated by discrepancies observed between the average result of previously existing questionnaires and results about the services obtained in other ways. Related to that, Jensen et al. point that the time interval, ranging from immediately after the service is provided to completion of questionnaires [10], significantly influences the perceived quality of provided service. The dynamics of end user attitudes is non-trivial and results obtained in different times cannot be readily compared [10]. Finally, Crow et al. [11] demonstrate that many means of measuring end user satisfaction, in the context of health services, do not show consumers' relative preferences for different attributes, even though such information is important for cost-effective decision-making.

\subsection{The problem}

This paper focuses on a company which provides to market the two qualitatively different services that are conducted with constant total daily available resources. The generic model of such a company is formulated and numerically simulated. The model incorporates in a tractable way the previously observed characteristics of end user dynamics, company's initiatives and responses.

In section two the corresponding model is described. Section three contains the results of simulations. Section four discusses the results obtained. Section five summarises the paper and provides the readers with concluding remarks and perspectives for a future work.

\section{MODEL}

\subsection{Elements of the model}

Elements of the model are the two services, the environment and the rules governing the service conduction. The environment represents the market, in the meaning of end users or the customers. Time is discrete and the dynamics is homogeneous in time. Time units are 
enumerated with index $k=1,2, \ldots$. Since time unit is arbitrary it may represent an hour, a day, a week or some other realistic time interval depending on the particular case.

\subsection{The services}

In order to grasp as much as possible of complexity of realistic market dynamics, yet to preserve tractability of results, we assume that a company offers two services to the market. The cases of a larger number of services provided introduce calculation complexity, yet they are not expected to bring about qualitatively new characteristics. Before proceeding, let us note that the very company's characteristics are implicitly assumed to make possible the explicitly stated characteristics of services, and are otherwise unspecified. The simple service, $\mathrm{S}_{1}$, is conducted as one-action service, within a single time unit. The advanced service, $\mathrm{S}_{2}$, is conducted as two-action service, during two time units. Time interval between actions in the advanced service, $\tau$, is equal for all the end users, and constant in simulation, with $\tau=1,2, \ldots$. The company conducts a finite total number of actions in each time unit, denoted as $N$. During one simulation $N$ is a constant. Among these, in a $k^{\text {th }}$ time unit $N_{11}(k)$ actions are performed as $\mathrm{S}_{1}, N_{21}(k)$ actions are performed as first action of the advanced service $\mathrm{S}_{2}$, while $N_{22}(k)$ denotes number of performed second actions of the $S_{2}$. One has:

$$
N_{11}(k)+N_{21}(k)+N_{22}(k) \leq N
$$

For unique determination of these numbers two more parameters are introduced, denoted as the priority ratio and the urgency parameter. The priority ratio $\varphi$ is a ratio of actions belonging to the simple and to the advanced service:

$$
\varphi=\frac{N_{11}(k)}{N_{21}(k)+N_{22}(k)}
$$

Value $\varphi>1$ means that more actions are conducted for $\mathrm{S}_{1}$, thus that action has higher priority than $\mathrm{S}_{2}$, and conversely for $\varphi<1$.

The urgency parameter is a ratio of number of initial and final action of the advanced service, denoted as $\pi$.

$$
\pi=\frac{N_{21}(k)}{N_{22}(k)}
$$

Value $\pi<1$ implies that it is more important to finish previously started service within a prescribed time interval than to start a new one. Conversely, $\pi>1$ means that it is of higher priority to conduct first action when needed, while ending of $\mathrm{S}_{2}$ could be more diffuse.

Parameters $\varphi$ and $\pi$ are relevant for cases with market demand larger than $N$, in which cases these parameters enable the company to organise consistently the service conduction. Conversely, in cases when the company offers more actions related to services than the market needs, sign $>$ in Eq. (1) is relevant, denominators in Eqs. (2) and (3) may become equal to zero thus these expressions become useless. Combination of these two parameters defines a set of two-parameter strategies of functioning of a company within some market. One may argue that by varying (e.g. in different simulations, or within a single simulation) the values of these parameters the company puts, temporary or constantly, the priority on either the one or to the other service. Exploring possible outcomes of applying all admissible twoparameter strategies is out of the scope of this paper. Instead, we concentrate here on one stationary, rather simple strategy in which $\varphi$ and $\pi$ are constant during one simulation.

While notation $N_{11}$ seems somewhat redundant, it is nevertheless used throughout this paper in order to makes easier possible further generalisations onto an arbitrary number of services with different number of corresponding actions. 
In a simulation we consider $N$ as a constant, which means that either the company really keeps the $N$ constant, or that it changes $N$ in a time interval which is larger than time interval spanned by simulations.

\subsection{The market}

Market, or the environment, is modelled implicitly. We assume that its population is effectively infinite, and that internal dynamics of market consists of a large number of unspecified, yet mutually independent processes. As a result, we consider that in each time unit $n_{1}$ agents ask for $\mathrm{S}_{1}$ and $n_{2}$ agents ask for $\mathrm{S}_{2}$. These numbers are random variables assumed to follow Poisson, rare events distribution. Means of the corresponding distributions, $\mu_{1}$ and $\mu_{2}$, are assumed constant in the simulation. It is consistent with the assumption that total population of the environment is practically infinite. We parameterise mean values $\mu_{1}$ and $\mu_{2}$ by adaptation and frequency parameters. We represent these parameters as the total number of population asking for a service in some time unit, $\alpha$, and as their ratio, $\omega$, respectively:

$$
\begin{gathered}
\alpha=\mu_{1}+\mu_{2} \\
\omega=\frac{\mu_{1}}{\mu_{2}}
\end{gathered}
$$

The meaning of Eq. (4) is as follows. Ratio $\alpha / N$ denotes how the company adapted its structure and internal dynamics to the total market demand: the closer that ratio to 1 the more adapted the company. It is so because, on the one hand $\alpha / N \gg>1$ implies that the company can fulfil only a minute part of total market demand for services. This is a typical case in highly competitive market with a lot of companies offering similar or identical services. On the other hand, $\alpha / N<<1$ implies that a company invested too large resources in providing a services. The company's resources are thus prevalently unused and the perspective is that the company reallocates them into more useful resources. For completeness, let us also state that $\alpha / N \approx 1$ refers to the adapted company with possibly optimal allocation of its resources. If $\omega \gg>1$ then the market demand for $S_{1}$ is considerably larger than the market demand for $S_{2}$, conversely for $\omega<<1$. While in specific cases one usually has some relation between the intervals of achievable values of $\pi$ and of $\omega$ in this paper we consider these and all other parameters to be completely mutually independent.

\subsection{The dynamics}

Quantities introduced for tracing the model dynamics are number of end users who wait for some of the services in a given time interval: $w_{11}$ is the number of agents waiting for $\mathrm{S}_{1}, w_{21}$ is the number of agents waiting for the first action of $\mathrm{S}_{2}$ and $w_{22}$ is the number of agents waiting for the second action of $\mathrm{S}_{2}$.

The following three constitutive relations relate the number of agents who wait:

$$
\begin{gathered}
w_{11}(k)=w_{11}(k-1)+n_{1}(k)-N_{11}(k) \\
w_{21}(k)=w_{21}(k-1)+n_{2}(k)-N_{21}(k) \\
w_{22}(k)=w_{22}(k-1)+n_{21}(k-\tau)-N_{22}(k)
\end{gathered}
$$

with $k>\tau \geq 1$ and the initial conditions $w_{11}(1)=w_{21}(1)=w_{22}(1)=0$.

Finally, cumulative time averages $\bar{w}$, and corresponding cumulative standard deviations $s$, of Eqs. (6) to (8) are evaluated as follows: 


$$
\begin{gathered}
\bar{w}_{i j}(T)=\frac{1}{T} \sum_{k=1}^{T} w_{i j}(k) \\
s_{i j}=\frac{1}{T} \sum_{k=1}^{T} w_{i j}^{2}(k)-\left[\bar{w}_{i j}(T)\right]^{2}
\end{gathered}
$$

with $i j$ denoting pairs 11, 21 and 22. Time interval for averaging, $T$, starts simultaneously with simulation and not after some finite time unit $k_{0} \gg>1$ passed. In that sense cumulative values are relevant for $T>>1$ so that influence of initial conditions becomes minute.

In order to suppress random variations caused by Poisson distributions underlying $n_{11}$ and $n_{21}$, for each set of parameters simulations were run $M$ times and corresponding average and variation of relevant quantities are shown. We refer to quantities obtained in such a way as to the ensemble average and ensemble variation, respectively:

$$
\begin{gathered}
w_{i j}^{(E)}(T)=\left.\frac{1}{M} \sum_{r=1}^{M} \bar{w}_{i j}(T)\right|_{r} \\
s_{i j}^{(E)}(T)=\frac{1}{M} \sum_{r=1}^{M}\left[\left.\bar{w}_{i j}(T)\right|_{r}\right]^{2}-\left[w_{i j}^{(E)}(T)\right]^{2}
\end{gathered}
$$

with superscript $(E)$ denoting the ensemble related quantity, and $\left.\right|_{\mathrm{r}}$ meaning that quantity to the left of the vertical bar is evaluated in the $r^{\text {th }}$ run, with $r=1, \ldots, M$.

We took $N=100$ throughout simulations. Because of the generic character of the model, that prescription does not introduce further loss of the generality as it covers all qualitatively different cases. A unique simulation is obtained with prescribed Eqs. (1) to (5) and $\tau$.

\section{RESULTS}

Simulations were performed for time scales ranging from 100 to 10000 . For simplicity, duration of simulations in results shown was fixed at 800 time units. Qualitative characteristics of model dynamics were clearly revealed in that duration.

Adaptation parameter $\alpha$ was taken equal to $0.1 \cdot N, N$ and $10 \cdot N$ in simulations. Listed values cover the cases of non-adapted, adapted and again non-adapted company, respectively. Cases $\alpha=0.1 \cdot N$ and $\alpha=10 \cdot N$ bring about trivial dynamics: if $\alpha=0.1 \cdot N$ then the values of Eqs. (6) to (8) are most of the time equal to zero, with rare, isolated and short-lasting deviations from zero caused by extremely rare, large numbers of $n_{11}$ or $n_{21}$. In the opposite extreme, $\alpha=10 \cdot N$, values of Eqs. (6) to (8) steadily rise in time without limit. The simulations revealed that a qualitative change in market dynamics of a company occurs for $\alpha \approx 0.6 \cdot N$ which we graphically represent and further analyse in details.

Frequency parameter $\omega$ was taken equal to $0.1,1$ and 10 in various simulations. However, values $\omega=0.1$ and $\omega=10$ bring about trivial dynamics as they effectively mean that majority of dynamics is linked to a single service, with minute influence of the other service. Because we want to exploit dynamics with two services of comparable influence, in simulations we considered $\omega \approx 1$, while results are shown for $\omega=1$.

The priority ratio (2) significantly influences Eqs. (6) to (8). In general, for $\varphi<1$ there are more end users waiting for $\mathrm{S}_{1}$, conversely for $\mathrm{S}_{2}$. However, time span of fluctuations of Eqs. (6) to (8) considerably changes with changes in $\varphi$.

Changes in urgency parameter (3) does not influence significantly a portion of Eq. (6) in total number of end user who wait, so we present simulations with fixed $\pi=1$. As a consequence, there was virtually no end users who wait for the second action of $\mathrm{S}_{2}$ : 
$w_{22}(k) \approx 0$, thus the dynamics simplified to interplay between $w_{11}$ and $w_{21}$. Finally, $\tau$ was taken two orders of magnitude smaller than $T$.

Fig. 1 shows typical time dependence of numbers of end users who wait for characteristic $\varphi$ and $N$. Figs. 2 and 3 show time dependence of ensemble averages and ensemble standard deviations, respectively, of numbers of agents who wait. Fig. 4 shows how the ensemble averages of end users who wait, $w_{11}^{(E)}$ and $w_{21}^{(E)}$, depend on $\tau$, while Fig. 5 shows how the ensemble-taken quantities depend on the number of actions performed daily. Fig. 6 shows combined influence of $\varphi$ and $N$ onto ensemble averaged number of end users who wait for a service, for their given range. For smaller values of $\varphi$ and $N$ diverges the number of end users who wait, while for $\varphi>1$ there is a symmetrical situation regarding changes of $w_{11}$ and $w_{21}$.
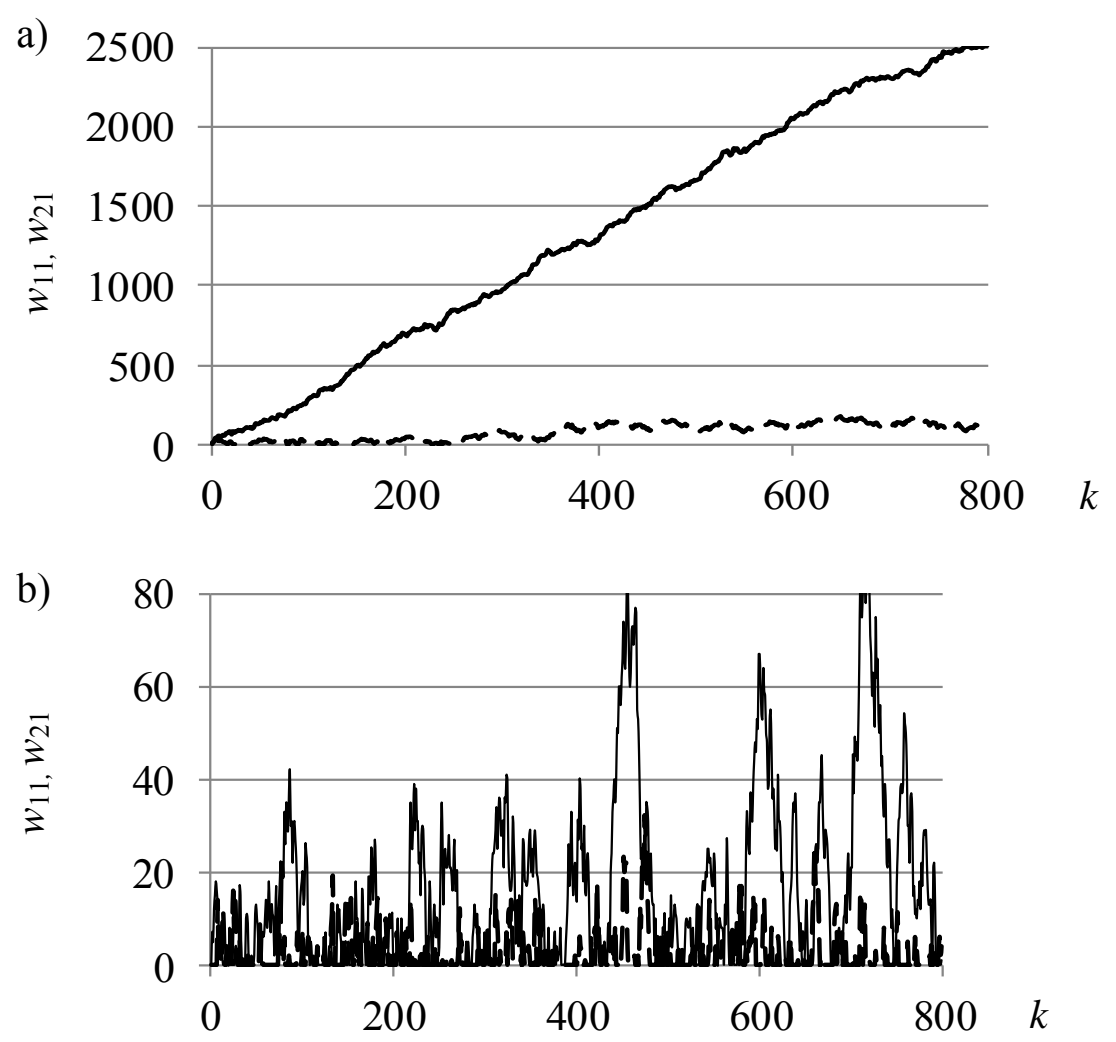

c)

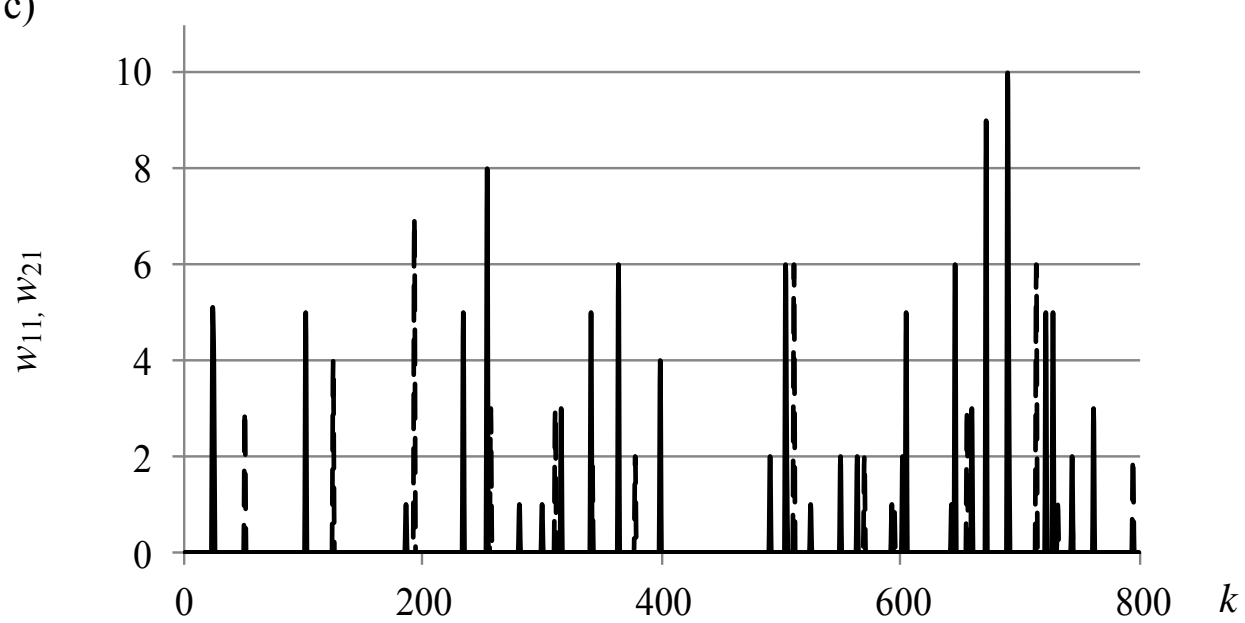

Figure 1 (the remaining part and complete title - see on the next page). 


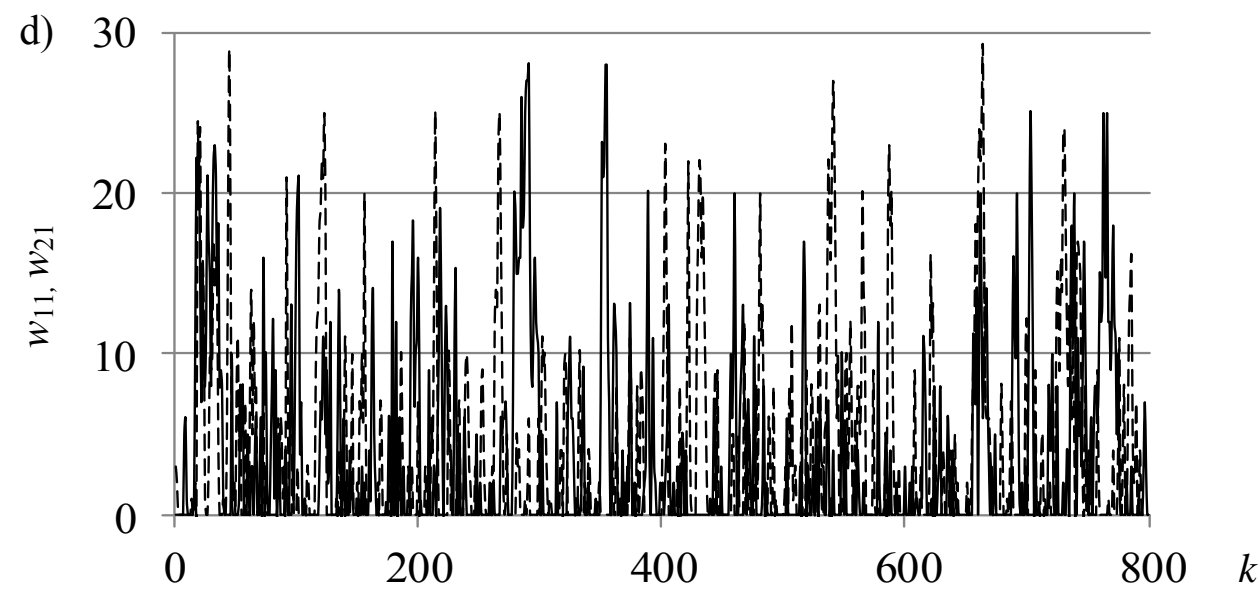

Figure 1: Time dependence of $w_{11}$ (full line) and $w_{21}$ (dashed line) with $\tau=10$ and for: a) $N=150$ and $\varphi=0.95$, b) $N=160$ and $\varphi=0.95$, c) $N=195$ and $\varphi=0.95$ and d) $N=160$ and $\varphi=1$.
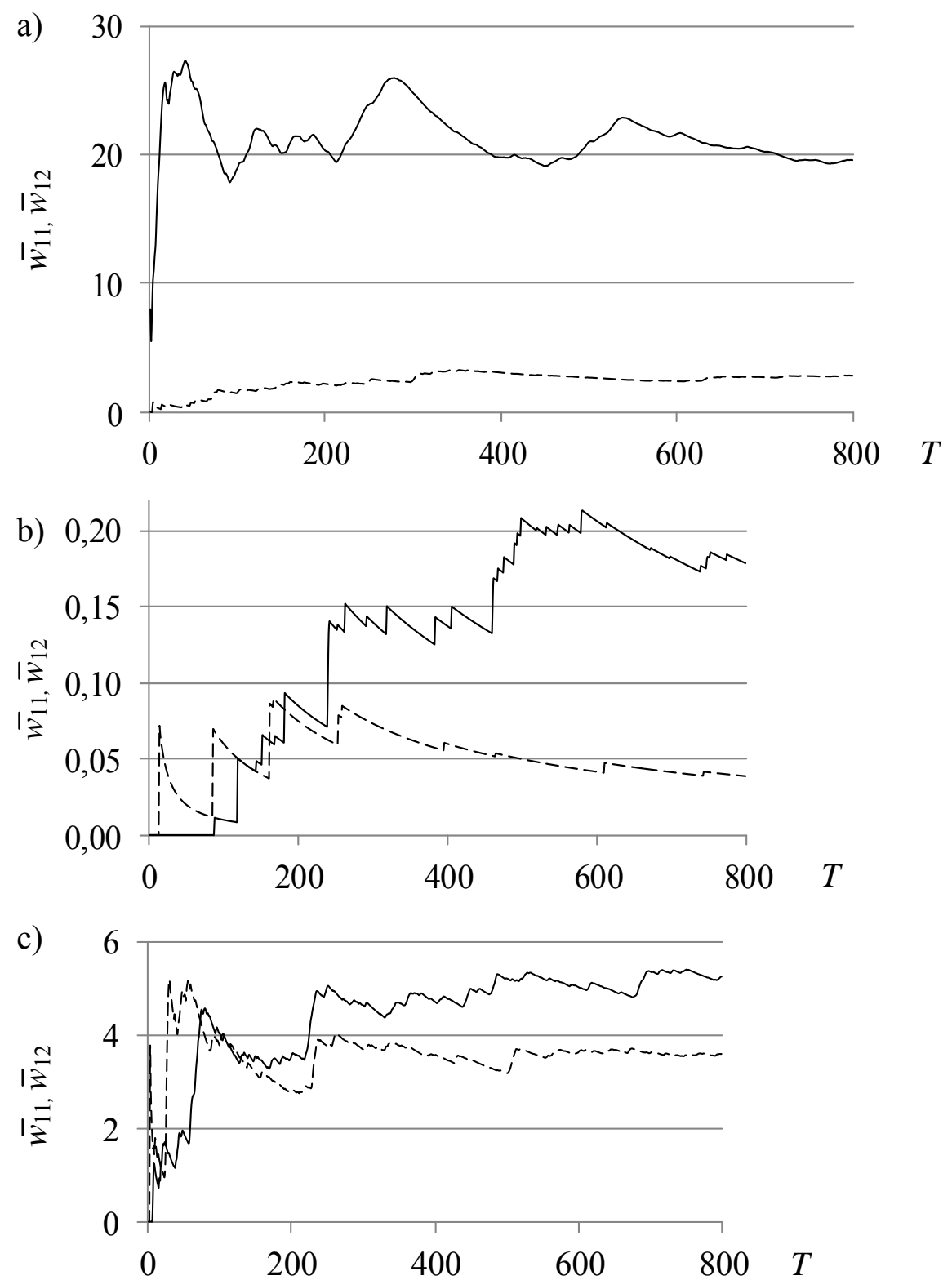

Figure 2: Dependence of averages $\bar{w}_{11}$ (full line) and $\bar{w}_{21}$ (dashed line) on averaging time $T$ with $\tau=10$ and for: a) $N=160$ and $\varphi=0.95$, b) $N=195$ and $\varphi=0.95$ and c) $N=160$ and $\varphi=1$. 

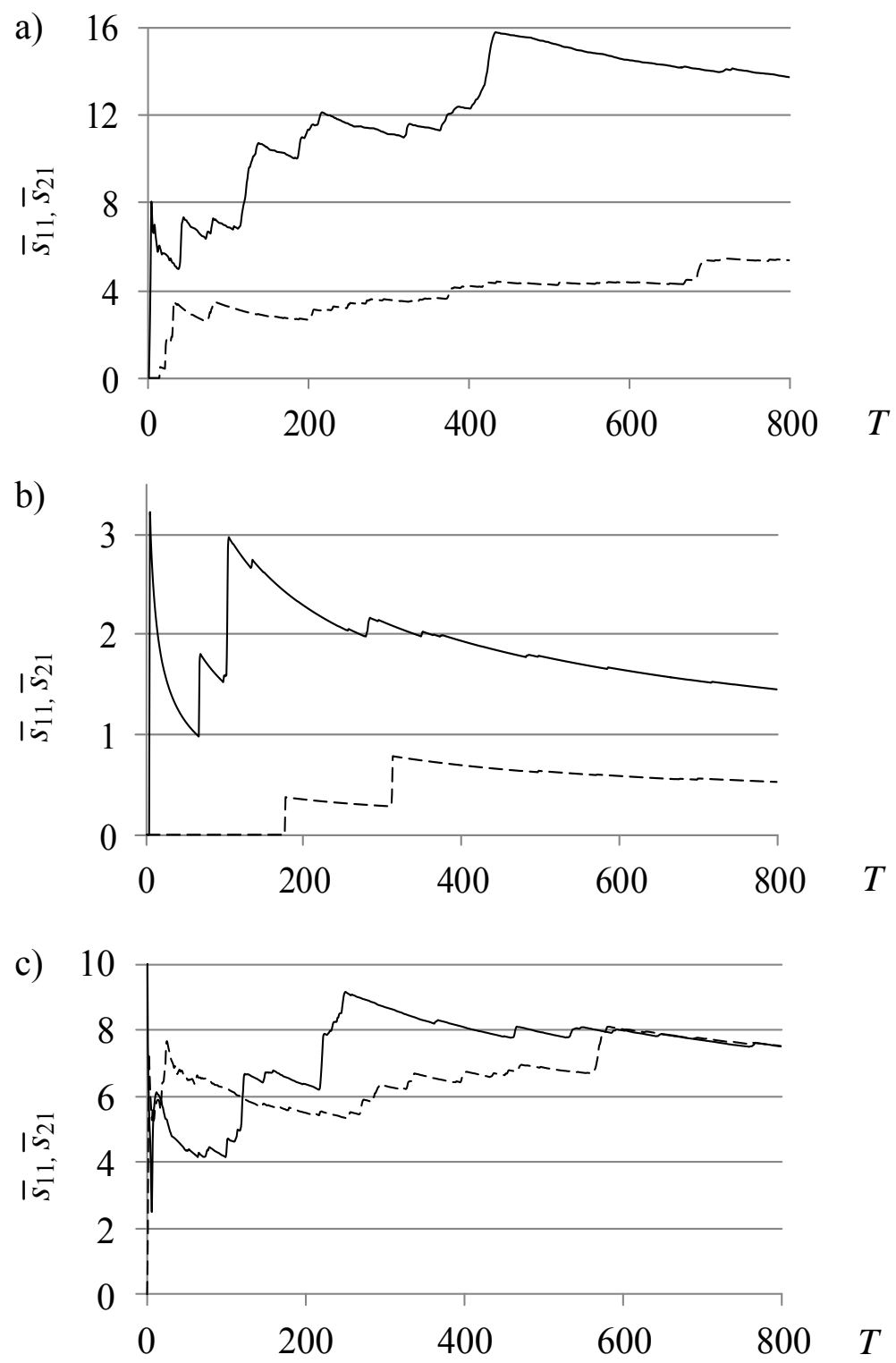

Figure 3: Dependence of standard deviations $\bar{S}_{11}$ (full line) and $\bar{S}_{21}$ (dashed line) on averaging time $T$ with $\tau=10$ and for: a) $N=160$ and $\varphi=0.95$, b) $N=195$ and $\varphi=0.95$ and c) $N=160$ and $\varphi=1$.

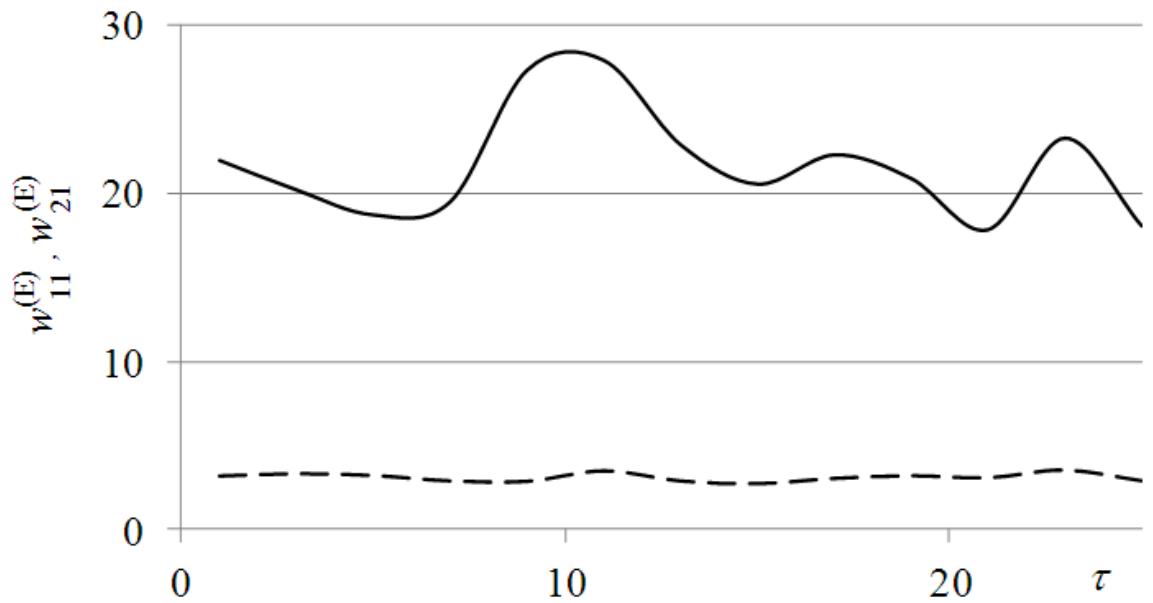

Figure 4: Dependence of ensemble averages $w_{11}^{(E)}$ (solid line) and $w_{21}^{(E)}$ (dashed line) on $\tau$ with $M=10, T=800, N=160$ and $\varphi=0.95$. 


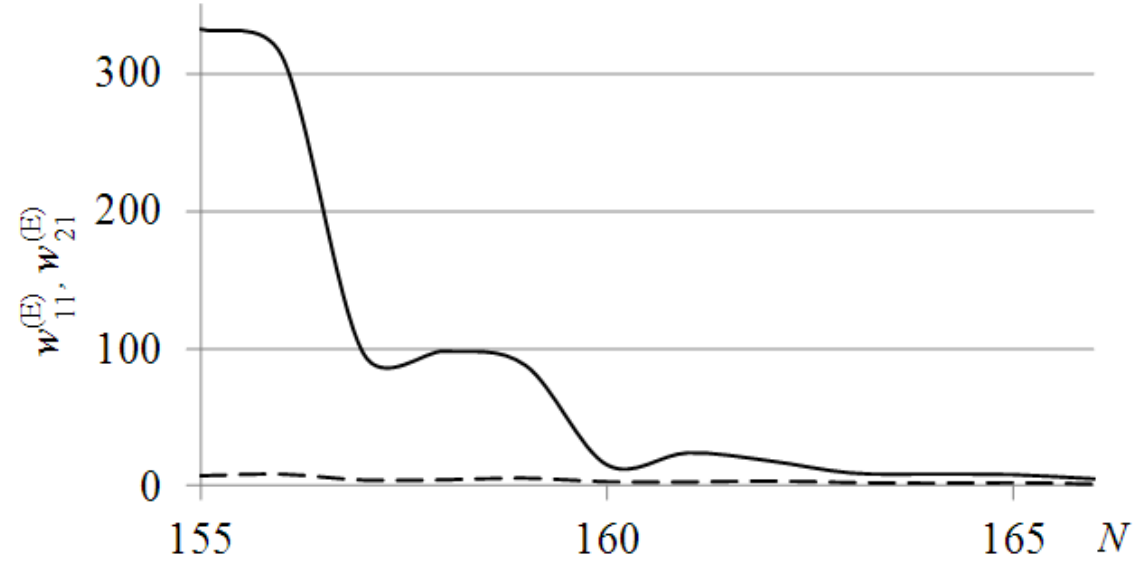

Figure 5: Dependence of ensemble averages $w_{11}^{(E)}$ and $w_{21}^{(E)}$ on $N$ for $\varphi=0.95, \tau=10$ and $M=5$.

a)

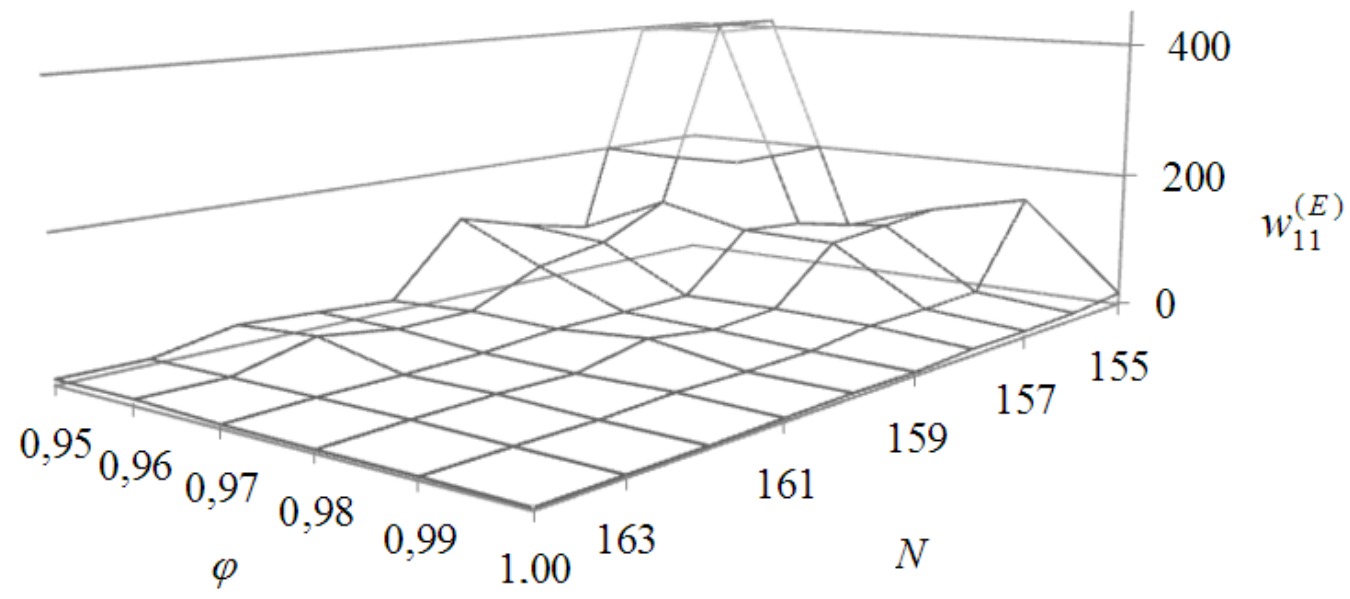

b)

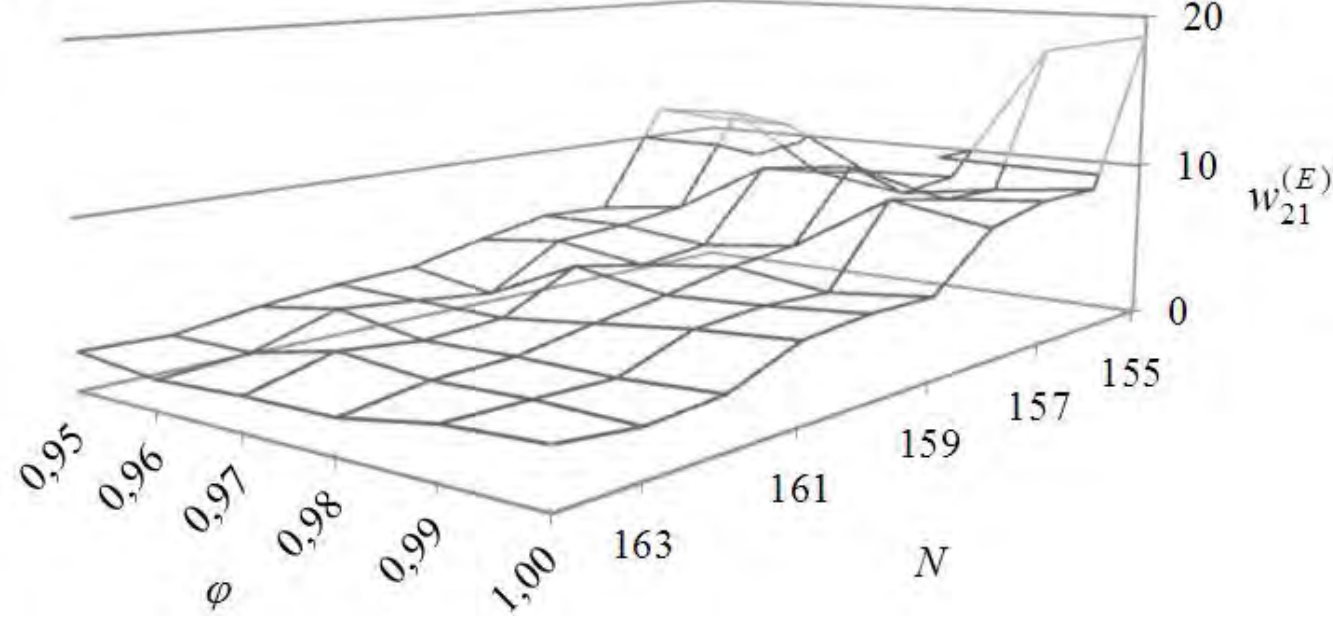

Figure 6: Dependence onto $\varphi$ and $N$ of ensemble averages: a) $w_{11}^{(E)}$, b) $w_{21}^{(E)}$; in both graphs $\tau=10$ and $M=5$. Note the different spans of the vertical axis.

\section{DISCUSSION}

Results reveal several different types of dynamics: diverging dynamics occurs for $\varphi$ rather different from 1 and stationary dynamics occur for $\varphi \approx 1$. In case $\varphi<1$ prevalently wait the 
end users who need $\mathrm{S}_{1}$, while in case $\varphi>1$ prevalently wait end users who need $\mathrm{S}_{2}$. It is, thus, sufficient to concentrate onto $\varphi<1$. Fig. $1 \mathrm{a}$ is an example of diverging dynamics in that the number of end users, who wait for a service, gradually rises in time with a finite stochastic contribution. In this case total number of daily conducted actions $N$ equals 150 . Fig. $1 \mathrm{~b}$ is an example of stationary dynamics obtained for $N$ somewhat larger than in Fig. 1 a: the number of end users who wait for their service is bounded. Dynamics is characterised with isolated and rapid rise in number of agents who wait for a service, followed by ceasing after several time units. Maximal values of numbers of agents who wait are, nevertheless, considerably smaller than corresponding numbers in the same time unit in case shown in Fig. 1 a. Similarly, Fig. $1 \mathrm{c}$ shows that in case $\alpha \approx 0.5 \cdot N$ stochastically induced bursts of number of agents who wait their services are limited to a single time unit or few of them. Comparing Figs. $1 \mathrm{~b}$ and $1 \mathrm{~d}$ one may argue that in cases when $\varphi$ rises to 1 local extrema, of number of agents who wait, are bounded by smaller and smaller values, are more equally distributed among $w_{11}$ and $w_{21}$ and are of shorter duration.

Similar conclusions can be reached starting from Fig. 2. In particular, Fig. 2 b shows that the average values are approximately equal to zero for most of the simulation duration, and are characterised with gradual diminishing interrupted with sudden rises, caused by rare events of suddenly large number of end users asking for a service. That corresponds to sparse deviations of these numbers from zero as seen in Fig. 1 c. Fig. 2 a can be accompanied with the discussion similar to the discussion accompanying Fig. 2 b, with the difference in considerably larger values on the ordinate axis in Fig. 2 a. Finally, Fig. 2 c shows that relative importance of the number of agents, who wait for their services, fluctuates, so part of the simulation period $w_{11}>w_{21}$ and part of time conversely. Fig. 3 supports that discussion, since corresponding graphs show combination of gradual diminishing of the standard deviations, interrupted with sudden, finite rises.

Fig. 4 reveals the drastic, volatile dependence of the $w_{21}$ onto $w_{21}$ for $\tau$ time units before. The volatility is larger for larger absolute amounts of $w$-s, i.e. $w_{11}$ (which is larger because of $\varphi<1$ ) and considerably smaller for the lesser of $w$-s, here the $w_{21}$. Similar behaviour show curves for the corresponding standard deviations, which are not shown for conciseness. However, dependence on these ensemble averages onto the total number of actions conducted daily $N$ is somewhat smoother, Fig. 5. In particular, one can observe gradual change between the two types of dynamics, the diverging and the stationary dynamics. The change occurs in the interval of $N$ the width of which is approximately 3-4 \% of total $N$. It is to be noted that the change occurs for values of $N$ which are much larger than 100, the corresponding value of the sum of daily means of end users asking for services, $\mu_{1}+\mu_{2}$, as introduced in Eq. (4). Numbers of agents who wait depend on a combined value of $\varphi$ and $N$, for other parameters constant, Fig. 6. For larger $\varphi$ the change between the two types of dynamics moves to smaller $N$. That is practically important result as it means that by changing the priority level of services, one may lower the number of agents who wait for services, even if that brings about the smaller total number of daily conducted actions $N$. The underlying reasoning is that the observed dynamics of a company is rather non-optimised so the stated changes are one realisation of how it can be improved. That result is interesting for further researches as it points out the possibility of finding a non-trivial optimal performance of a company. Simultaneous taking into account the graphs in Fig. $6 \mathrm{a}$ and Fig. $6 \mathrm{~b}$ emphasises that, based on the span of $\varphi$ and $N$ for which changes in dynamics occur, the lowering of $w_{11}$ occurs without corresponding rise in $w_{21}$, and with rapid finishing of $\mathrm{S}_{2}$, i.e. with $w_{22} \approx 0$ in all cases. While that is valid for $\varphi$ slightly less than 1 , similar changes occur for $\varphi$ slightly larger than 1 , with replacement between $w_{11}$ and $w_{21}$. 


\section{SUMMARY AND CONCLUSIONS}

A generic model, of a company which conducts two qualitatively different types of services in a market, is formulated and its dynamics analysed. The model incorporates qualitatively, in a simplified and prevalently implicit way, different characteristics of realistic companies. The observed dynamics has several, qualitatively different types, which are related to the parameters describing the company's performance. The emphasis in the paper is put onto the stationary dynamics as it enables the company to function, in principle, indefinitely. Stationarity is linked to equalising the priorities of the services offered $(\varphi \rightarrow 1)$ and to sufficient adaptation of the company to its environment, i.e its market. But, the stationarity is highly non-uniform, and consists of two contributions intertwined in a random manner. One contribution is that of sudden and intense change in number of agents who ask for a service, while the other is that of gradual diminishing of large deviations in number of such agents. Differences in dynamics point to different levels of optimisation achieved and enable the researchers and practitioners to change the overall dynamics of a company without need for additional resources. Specifically, by equalising the priority of services (i.e. $\varphi \rightarrow 1$ ) one can lower the total number of end users who wait for services without raising the number of daily conducted services $(N)$, which in practice means without needs for additional resources.

There are aspects of the formulated model which asks for further research. Among these, we extract two about the company and two about the market. Regarding the company, first the detailed prescription how to form values of introduced parameters in Eqs. (2) to (5) is needed in order to make possible linking the simulation to a specific company. Secondly, while in this paper the parameters in Eqs. (2) to (5) are considered mutually independent, that assumption needs either the more rigorous proof, or the extraction of possible limitations. Regarding the market, it should be formulated as a competitive market, in which more than one company exists that provides the same set of services. Finally, in many situations one cannot expect that market demand fits the rare event distribution. Instead, other types of distributions needs to be incorporated in the model and corresponding stationary dynamics of the company analysed.

\section{ACKNOWLEDGEMENT}

This work has been supported in part by the Croatian Science Foundation under the project number IP2014-09-6963.

\section{REFERENCES}

[1] Epstein, J. M.; Axtell, R. L. (1996). Growing Artificial Societies, Brookings Institution Press \& MIT Press, Washington D.C.

[2] Baxter, N.; Collings, D.; Adjali, I. (2003). Agent-based modelling - intelligent customer relationship management, BT Technology Journal, Vol. 21, No. 2, 126-132, doi:10.1023/A:1024455405112

[3] Kaihara, T. (2003). Multi-agent based supply chain modelling with dynamic environment, International Journal of Production Economics, Vol. 85, No. 2, 263-269, doi:10.1016/S09255273(03)00114-2

[4] Runje, B.; Krstić Vukelja, E.; Horvatić, A. (2015). Comparison of different simulations methods in case of service-providing companies, Interdisciplinary Description of Complex Systems, Vol. 13, No. 3, 472-478, doi:10.7906/indecs.13.3.11

[5] Terano, T.; Naitoh, K. (2004). Agent-based modeling for competing firms: from balancedscorecards to multiobjective strategies, Proceedings of the $37^{\text {th }}$ Annual Hawaii International Conference on Systems Sciences, doi:10.1109/HICSS.2004.1265251 
[6] Acko, B.; Brezovnik, S.; Crepinsek Lipus, L.; Klobucar, R. (2015). Verification of statistical calculations in interlaboratory comparisons by simulating input datasets, International Journal of Simulation Modelling, Vol. 14, No. 2, 227-237, doi:10.2507/IJSIMM14(2)4.288

[7] Acko, B.; Sluban, B.; Tasič, T.; Brezovnik, S. (2014). Performance metrics for testing statistical calculations in interlaboratory comparisons, Advances in Production Engineering \& Management, Vol. 9, No. 1, 44-52, doi:10.14743/apem2014.1.175

[8] Crepinsek Lipus, L.; Matus, M.; Acko, B. (2013). Optimization of calibrating HeNe laser interferometers by sample-period simulation, International Journal of Simulation Modelling, Vol. 12, No. 3, 154-163, doi:10.2507/IJSIMM12(3)2.231

[9] Williams, B.; Coyle, J.; Healy, D. (1998). The meaning of patient satisfaction: An explanation of high reported levels, Social Science \& Medicine, Vol. 47, No. 9, 1351-1359, doi:10.1016/S02779536(98)00213-5

[10] Jensen, H. I.; Ammentorp, J.; Kofoed, P.-E. (2010). User satisfaction is influenced by the interval between a health care service and the assessment of the service, Social Science \& Medicine, Vol. 70, No. 12, 1882-1887, doi:10.1016/j.socscimed.2010.02.035

[11] Crow, R.; Gage, H.; Hampson, S.; Hart, J.; Kimber, A.; Storey, L.; Thomas, H. (2003). The measurement of satisfaction with health care: implications for practice from a systematic review of the literature, Health Technology Assessment, Vol. 6, No. 32, doi:10.3310/hta6320 